\title{
Abnormal expression profile of plasma- derived exosomal microRNAs in patients with treatment-resistant depression
}

\author{
Lian-Di Li ${ }^{1 \dagger}$, Muhammad Naveed ${ }^{1 \dagger}$, Zi-Wei Du ${ }^{1 \dagger}$, Huachen Ding ${ }^{2,3}$, Kai Gu$^{4}$, Lu-Lu Wei ${ }^{1}$, Ya-Ping Zhou', Fan Meng ${ }^{1}$, \\ Chun Wang ${ }^{2,3}$, Feng Han ${ }^{5^{*}}$, Qi-Gang Zhou ${ }^{1,4^{*}}$ and Jing Zhang ${ }^{1 *}$ (D)
}

\begin{abstract}
Whether microRNAs (miRNAs) from plasma exosomes might be dysregulated in patients with depression, especially treatment-resistant depression (TRD), remains unclear, based on study of which novel biomarkers and therapeutic targets could be discovered. To this end, a small sample study was performed by isolation of plasma exosomes from patients with TRD diagnosed by Hamilton scale. In this study, 4 peripheral plasma samples from patients with TRD and 4 healthy controls were collected for extraction of plasma exosomes. Exosomal miRNAs were analyzed by miRNA sequencing, followed by image collection, expression difference analysis, target gene GO enrichment analysis, and KEGG pathway enrichment analysis. Compared with the healthy controls, 2 miRNAs in the plasma exosomes of patients with TRD showed significant differences in expression, among which has-miR-335-5p were significantly upregulated and has-miR-1292-3p were significantly downregulated. Go and KEGG analysis showed that dysregulated miRNAs affect postsynaptic density and axonogenesis as well as the signaling pathway of axon formation and cell growths. The identification of these miRNAs and their target genes may provide novel biomarkers for improving diagnosis accuracy and treatment effectiveness of TRD.
\end{abstract}

Keywords: Depression, Exosomes, MicroRNAs, Biomarker, High-throughput sequencing

\section{Introduction}

Depression has been a common mental illness and major public health problem. Its core symptoms are characterized by low mood and anhedonia. Depression seriously reduces the efficiency of patients' work and quality of life and even leads to suicide. In 2008, the World Health Organization (WHO) listed major

\footnotetext{
* Correspondence: fenghan169@njmu.edu.cn; qigangzhou@njmu.edu.cn; zj1984@njmu.edu.cn

'Lian-Di Li, Muhammad Naveed and Zi-Wei Du contributed equally to this work.

${ }^{5}$ Key Laboratory of Cardiovascular and Cerebrovascular Medicine, School of Pharmacy, Nanjing Medical University, Nanjing 211166, Jiangsu Province, China

${ }^{1}$ Department of Clinical Pharmacology, School of Pharmacy, Nanjing Medical University, Nanjing 211166, Jiangsu Province, China

Full list of author information is available at the end of the article
}

depression as the third leading cause of the global burden of disease, and it is predicted that the condition will rank first by 2030 [1]. Although many researchers focus on this field, the understanding of the pathophysiology of depression is still limited. At present, the pathological mechanisms of depression mainly include the abnormal metabolism of serotonin (5-HT), norepinephrine (NE), and other monoamine neurotransmitters, hypothalamicpituitary-adrenal (HPA) axis hyperfunction, neuroinflammation, and impaired synaptic plasticity, etc. [2-5]. At present, selective serotonin reuptake inhibitors (SSRIs) are still widely used as first-line treatments for depression; however, $30 \%$ or more of patients fail to get response to pharmacologic interventions and have repeated depressive episodes, diagnosed as treatmentresistant depression (TRD) [6]. In recent years, the 
research on biomarkers of depression has become a new hotspot. Biomarker research would not only hold promise in individualized diagnosis and treatment, but also enhance treatment responsivity in TRD.

Exosomes are extracellular vesicles with a lipid bilayer and a diameter of $40-200 \mathrm{~nm}$. They are widely distributed in the blood, cerebrospinal fluid (CSF), urine, and other body fluids. Many cells in an organism, including neurons, can secrete exosomes [7]. The bilayer membrane structure of exosomes allows them to bind to the cell membrane and readily cross the blood-brain barrier (BBB) to regulate the function of the nervous system [8]. There are various biologically active molecules, including lipids, proteins, and small RNA in exosomes, which could be transported to nearby or distant cells. Exosomes released by the invagination of intracellular lysosomal micro-particle in the central nervous system (CNS) may play an active role in the occurrence or development of diseases in the CNS such as Alzheimer's disease (AD), Parkinson's disease (PD), prion disease, multiple sclerosis (MS), schizophrenia (SCZ), and brain tumors [9]. The exosome translocation between cells participates in the communication between cells and regulates the function of target cells. A large number of studies have shown that exosomes play an important role in the communication of the CNS, neural regeneration, synaptic plasticity, and neuroinflammation [1012].

MicroRNA is a small non-coding RNA, about $22 \mathrm{nu}$ cleotides in length, which can directly interact with the seed complementary sequence in the $3^{\prime}$ untranslated regions ( $3^{\prime}$-UTR) of the target messenger RNA (mRNA), thus inhibiting the expression of the target gene. Compared with other biological fluids such as plasma and saliva, many miRNAs exist in exosomes [13]. The lipid bilayer structure of exosomes could protect miRNAs, making them more stable than free miRNAs. Many studies have shown that miRNAs is widely involved in regulating various systems, including the nervous system, and may play a vital role in brain function, especially in neurogenesis, neuronal development, and synaptic plasticity [14]. Studies have shown that miRNAs expression in exosomes can be changed in different disease states, making the miRNAs the candidates as the biomarker for the pathogenesis of CNS diseases [15].

In view of the regulatory role of exosomes in neuroinflammation, neurogenesis, and plasticity, exosomes have long been hypothesized to be involved in the psychopathological processes of mental disorders. Banigan et al. detected miRNAs changes in exosomes in frozen postmortem prefrontal cortices of patients with $\mathrm{SCZ}$ and bipolar disorder and found that microRNA-497 (miR-497) increased in SCZ patients and miR-29c upregulated in bipolar patients [16]. Du et al. conducted the genome- wide miRNA expression profile analysis on serumderived exosomes from 49 first-onset, drug-free SCZ patients, and 46 normal persons and found 11 miRNAs showed a significant difference between the two groups, which can be used to classify samples from SCZ patients and control subjects with nearly $90 \%$ accuracy in the training samples, and approximately $75 \%$ accuracy in the testing samples [17]. Recently, some researches have indicated that the exosomes and associated miRNAs may contribute to the inflammatory mechanisms underlying depression [18]. The exosome-derived miR-139-5p had the potential to be a biomarker for major depressive disorder [19]. Furthermore, studies have shown that exosomes from patients with major depression cause depression-like behaviors in mice involved in miR-1395p-regulated neurogenesis [20]. Bone marrow mesenchymal stem cell-derived exosomes upregulated miR-26a to boost hippocampal neuron proliferation and suppressed apoptosis in depressed rats [21], and NK cell-derived exosomes could carry miR-207and alleviate depressionlike symptoms in mice [22]. These results indicate that exosomes are involved in the occurrence and development of depression and other mental disorders. However, the potential role of exosomes in the pathophysiology of treatment-resistant depression (TRD) is still poorly understood. Given that exosomes are involved in psychopathological processes, including neuroinflammation, neurogenesis, plasticity, and epigenetic regulation [23], exosomes are likely to play an important role in the pathogenesis of depression.

This study collected the peripheral blood samples from 4 TRD patients and 4 healthy controls and extracted miRNAs for high-throughput sequencing also known as next-generation sequencing (NGS). We try to find the different major miRNAs in the plasma exosomes of TRD patients and their function in different signal pathways. The recognition of these miRNAs and their target genes may provide a new high-throughput screening platform for the early detection of the severity of depression and the treatment response, which will improve the diagnosis accuracy and treatment effectiveness of the depression.

\section{Materials and methods \\ Study participants}

The inclusion criteria for the patient with TRD were as follows [24]: (1) 18-65 years old; (2) recent diagnosis of depression by ICD-10 criteria for a mood disorder and depressive episode; (3) diagnosis of antidepressant resistance: recurrent attacks in recent 3 years after two or more depressants treatment; (4) no history of taking any other drugs; and (5) the total score of the Hamilton Depression Scale (HAM-D) was higher than 17, indicating mild to moderate depression. Before treatment, the patients were re-evaluated with HAM-D. At the same time, 
blood samples were collected simultaneously with symptom self-evaluation and before treatment. Subjects in the healthy control group matched the age of patients with depression; they (1) had no history of depression, (2) had a HAM-D score of 0-4 for more than 8 weeks, and (3) exclude any of the conditions described in the criteria. Participants (healthy controls and depressed patients) were excluded from the study if they had severe personality disorders, risk of suicide, pregnancy, thyroid disease, use of any psychotherapy, and participation in any other psychiatric intervention study.

This study was approved by the Nanjing First Hospital Organization evaluation committee, showed in supplementary information. All eight participants provided informed consent before signing up.

\section{Sample collection}

Plasma samples of depression patients and healthy control participants were collected before treatment. At least $4 \mathrm{ml}$ of venous blood was collected from the subjects' tubes with EDTA anticoagulant. After $10 \mathrm{~min}$ of centrifugation at $3000 \mathrm{rpm}$ at room temperature, plasma samples (ideally $2 \mathrm{ml} / \mathrm{sample}$ ) are stored in RNase antifreeze tubes at $-80^{\circ} \mathrm{C}$.

\section{Exosome isolation and identification}

Exosomes were isolated using ExoEasy Maxi Kit (Qiagen, 76064, USA). Briefly, $2 \mathrm{ml}$ plasma samples filtered using a $0.8 \mu \mathrm{m}$ filter syringe (Millipore, SLAA033SB, USA) are mixed with Buffer XBP and bound to an exoEasy membrane affinity spin column. The bound exosomes are washed with buffer XWP, eluted with $400 \mu$ l Buffer XE (an aqueous buffer containing primarily inorganic salts). The exosome samples were stored at $-20^{\circ} \mathrm{C}$ until further detection. Exosomes were observed by transmission electron microscopy (TEM) (FEI, Tecnai Spirit TEM T12). The size and concentration of the exosomes were assessed using Flow NanoAnalyzer (NanoFCM, Xiamen, China). Exosomal proteins CD81 (BD, 551108, USA) and CD9 (BD, 555317, USA) expression were measured using Nano-Flow analysis (NanoFCM, Xiamen, China).

\section{Exosome RNA extraction}

According to the manufacturer's instructions, using exoRNeasy Midi Kit (Qiagen, 77144, USA), separate the exosome RNA, including miRNA. In brief, exosome samples are homogenized in QIAzol Lysis Reagent, chloroform is added to the QIAzol eluate, and the aqueous phase is recovered and mixed with ethanol. Total RNA, including miRNA, binds to the spin column, washed three times, and eluted. Use Agilent 2100 Bioanalyzer (Santa Clara, CA, USA) to analyze the yield and quality of the separated RNA (Supplement figure 1).

\section{Next-generation sequencing analysis}

RNA fragments containing 17-30 nucleotides, or 15-35 nucleotides, were isolated by gel purification. A random primer is attached to both ends of the isolated RNA fragment and then reverse transcribed into the CDNA as previously described [25]. Amplification was carried out by PCR to establish a sequence library, and Illumina HiSeq highthroughput sequencing was carried out using the SE50 strategy [25]. The expression level of miRNA was obtained by Qiagen online platform. Edger package [26] was compared to those in the genome to get information about the location of known mature and precursor miRNAs in the genome. Clean reads in the genome were matched to the location of miRNA [27] and further analyzed its expression, sequence, and structure. As mentioned above, the target genes that differentially expressed miRNAs are predicted and sought by miRanda (http://www.microrna.org/ microrna/home.do), RNAhybrid (http://bibiserv.techfak.unibielefeld.de/rnahybrid/), and miRDB (http://www.mirdb. org/) database. The target genes of differentially expressed miRNAs were subjected to gene ontology (GO) and Kyoto encyclopedia of genes and genomes (KEGG) analyses as previously described [28, 29]. Gender-specific and sex hormone-regulated miRNAs were excluded from the study.

\section{Statistical analysis}

Unpaired Student's $t$ test with Welch's correction was used to estimate the differences between groups. All statistical analysis is performed by using primer 8 software (Cary, NC, USA). When the $p$-value is less than 0.05 , these results are considered significant.

\section{Results}

\section{Identification of extracted plasma exosomes}

As shown in Fig. 1, the exosomes are round or oval vesicles with different sizes $(30-100 \mathrm{~nm})$ under the electron microscope, which are composed of the outer membrane with deeper staining and the inner layer with shallow staining and lower electron density by the description of exosomes reported in the literature [30]. The size of exosomes ranged from 71 to $79 \mathrm{~nm}$, and the expression of CD9 and CD81 was positive.

\section{Identification of abnormally expressed miRNAs in patients with depression by NGS}

The study included four patients with major depressive depression and four healthy controls. Using high NGS, the analyzed miRNAs are considered candidate miRNAs if they are upregulated or downregulated $\geq 2$ times, and the adjusted $p$-value was $<0.05$. As shown in the heatmap of differential miRNAs expression in Fig. 2, there were 10 upregulated miRNAs: has-miR-19a-3p, hasmiR-144-3p, has-miR-130a-3p, has-miR-335-5p, hasmiR-101-3p, has-miR-1277-5p, has-miR-15a-5p, has- 

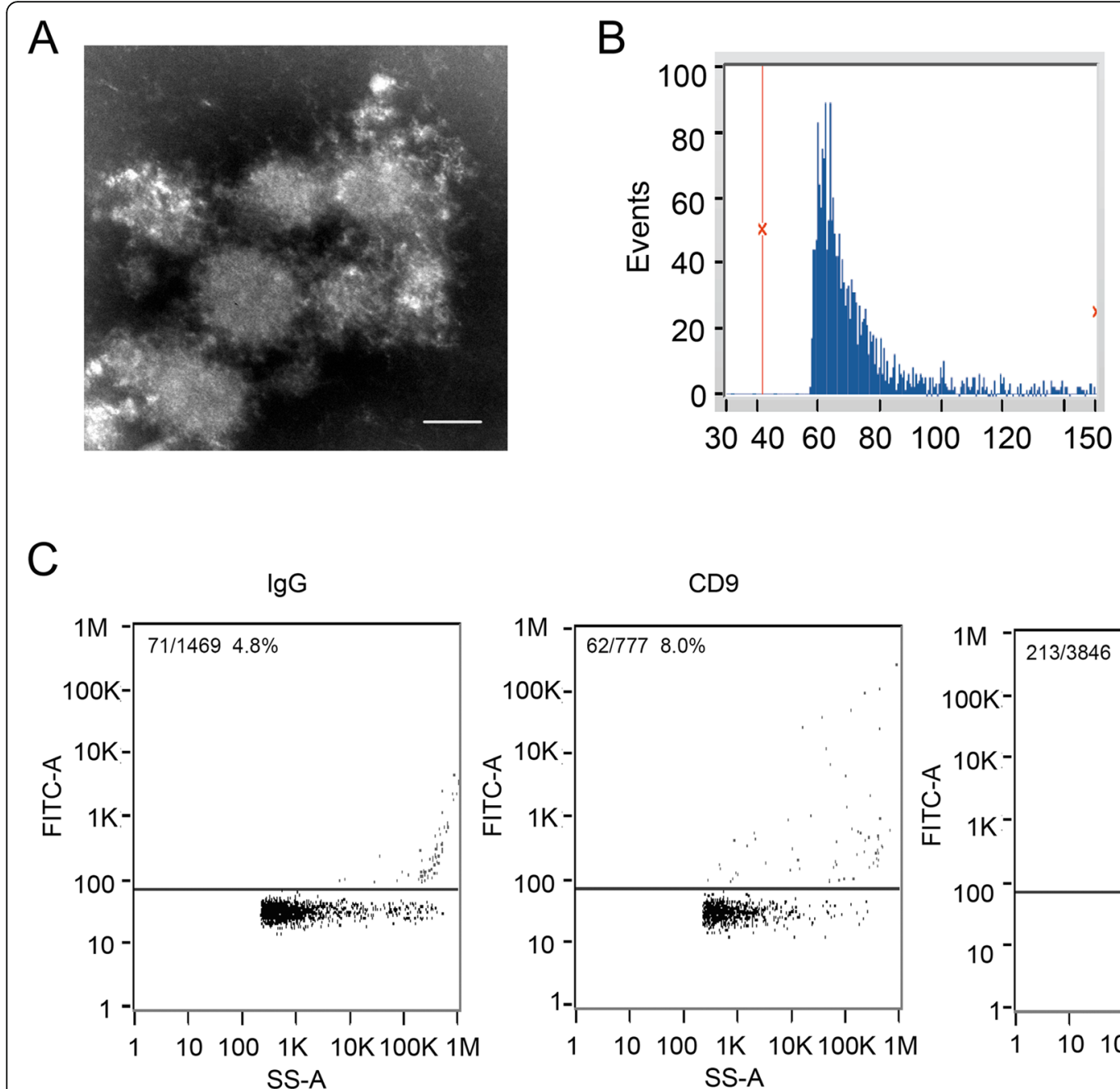

Total Events 2121

Gating Events 2003

$\%$ of all 94.44

Median $66.25 \mathrm{~nm}$

Mean $71.47 \mathrm{~nm}$

Std Dev. $15.70 \mathrm{~nm}$
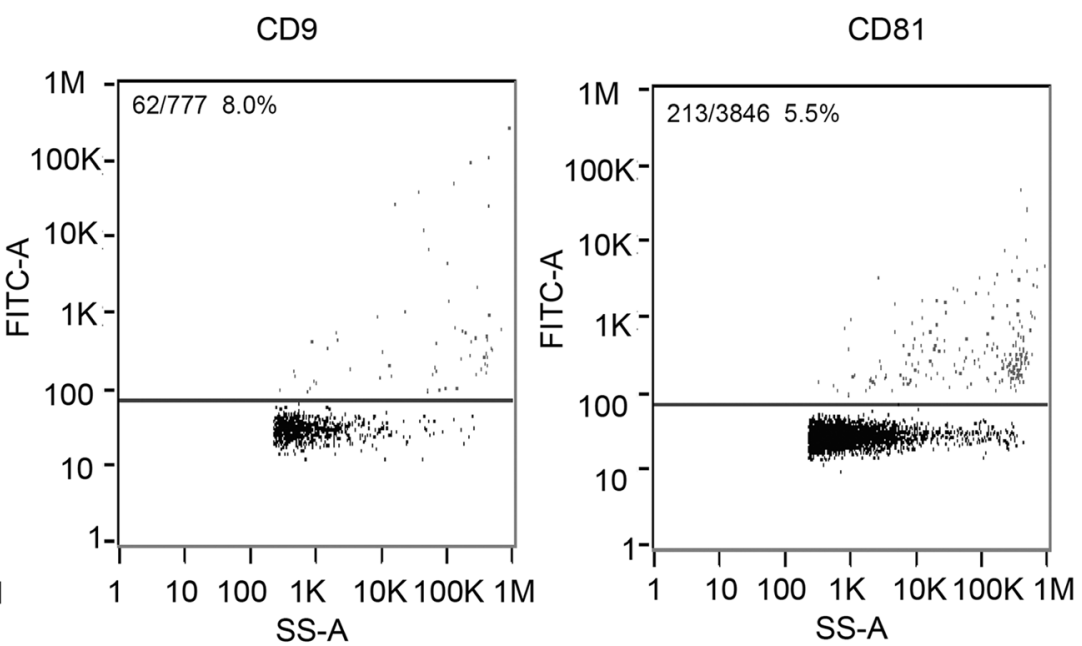

Fig. 1 The morphology and characteristics of extracted exosomes from patients with TRD and health controls. A The transmission electron micrograph of plasma-derived exosomes. Scale bar: 100nm. B The particle size distribution of plasma-derived exosomes. C The fluorescence of exosomal proteins (CD9 and CD81) was detected by flow cytometry

miR-29c-3p, has-miR-19b-3p, and has-miR-21-5p and 5 down-regulated miRNAs: has-miR-7704, has-miR-12923p, has-miR-1909-5p, has-5001-5p, and has-miR-4688 were identified by NGS analysis (Supplementary Table 1). We further used unpaired Student's $t$ test with Welch's correction to analyze the expression levels of these 15 microRNAs and found that has-miR-335 and has-miR-1292 still had statistical differences (Table 1). Therefore, we selected has-miR-335 and has-miR-1292 as candidate microRNAs for subsequent analysis.

Adjusted $p$-values were determined using unpaired Student's $t$-test with Welch's correction

\section{Analysis of differentially expressed miRNAs targeted genes}

Based on the 2 miRNAs with significant expression differences, all target genes in miRanda, RNAhybrid, and miRDB database were predicted $[31,32]$.
GO analysis of miRNAs differentially expressed in exosomes of patients with depression

Gene ontology (http://www.geneontology.org/) functional enrichment analysis of miRNAs target genes can show the functional enrichment of miRNAs target genes and clarify the differences among samples at the gene function level. As shown in Table 2, GO analyses the differentially expressed miRNAs are related to cell composition, biological process, and molecular functions. Cell composition includes the regulation of postsynaptic density and transcription regulators. Biological process includes the axonogenesis. The regulation of molecular functions consists of the post-transcriptional change.

The overlap value indicates the number of predicted downstream target genes of the candidate miRNAs identified versus the total number of genes related to the term in the GO gene expression network 


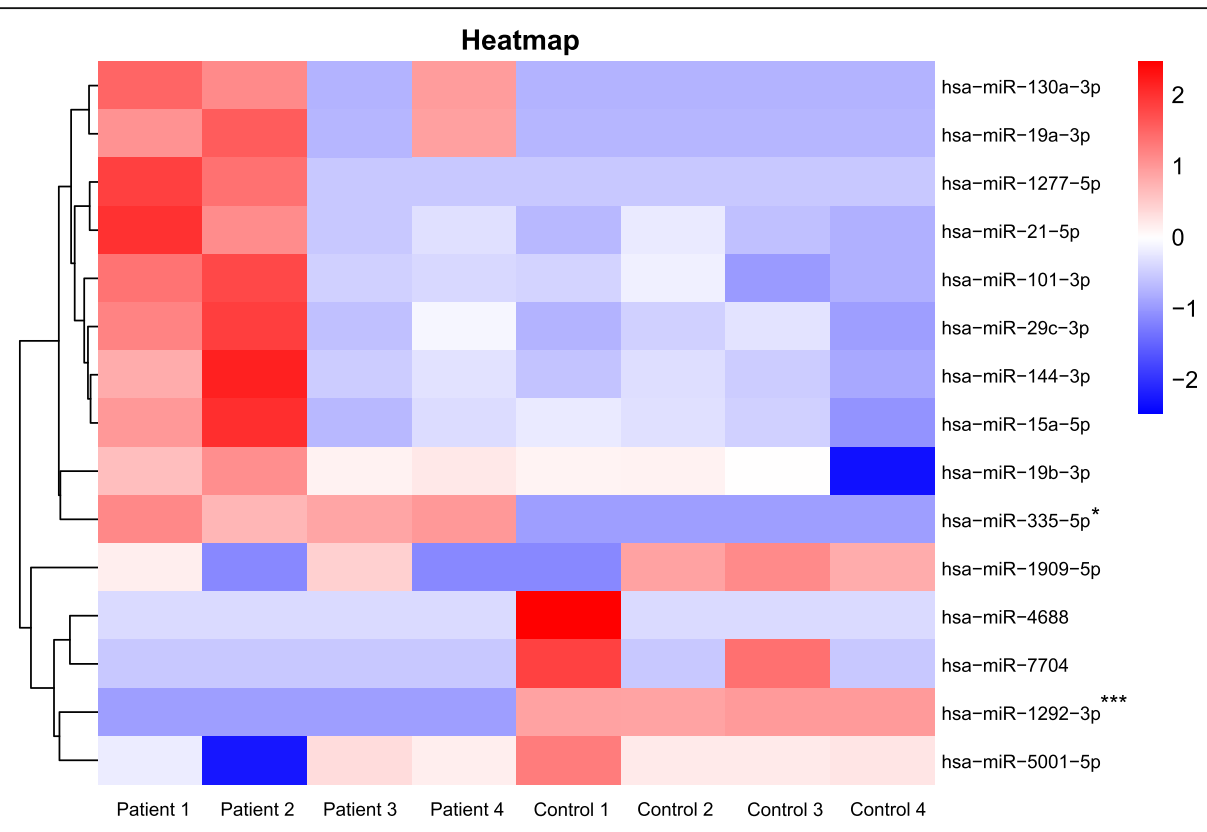

Fig. 2 Heatmap of differentially expressed miRNAs. This figure shows the distribution of 15 different miRNAs in four depression patients and four healthy controls. Values below 0 are superimposed in blue, and the smaller the number, the darker the color. Values above 0 in red and the larger the value, the darker the color. ${ }^{*} P<0.05,{ }^{* *} P<0.001$, unpaired Student's $t$-test with Welch's correction

\section{Enrichment KEGG analysis of differentially expressed miRNA target gene}

KEGG database is a knowledge base for systematic analysis of gene function, an association of genome information and functional information. As shown in Table 3, KEGG analysis of the differentially upregulated and downregulated expressed miRNAs revealed some are related to MAPK, Ras, and PI3K-AKT signaling pathway.

The overlap value indicates the number of predicted downstream target genes of the candidate miRNAs identified versus the total number of genes related to the term in the KEGG gene expression network

\section{Target gene regulated downstream signaling pathway of} miRNAs differentially expressed miRNAs

miRanda, RNAhybrid, and miRDB were used to predict different miRNAs target genes, and 1086 target genes were selected as the highest related target genes (Fig. 3). We used overlapping parts from two or more databases as predictive genes and then further searched the literature to select target genes related to depression. As shown in Table 4, 14 genes were selected as target genes.

Table 1 Significantly dysregulated miRNAs in plasma exosomes in TRD patients compared with healthy controls

\begin{tabular}{llll}
\hline MicroRNA & Fold-change & Up/down & Adjusted $\boldsymbol{p}$-values \\
\hline Has-miR-335-5p & 149.231458 & Up & 0.0315 \\
Has-miR-1292-3p & 0.007057 & Down & 0.0006 \\
\hline
\end{tabular}

These target genes involve the synaptic synthesis and transport of many neurotransmitters, including dopamine, glutamate, norepinephrine, serotonin, and the binding process with corresponding receptors.

\section{Discussion}

Exosomes are tiny vesicles secreted by cells. When exosomes were first found, they were considered as a way to excrete waste [45]. With the development of research, it was found that the mRNA and miRNA carried by exosomes can be translated into proteins in the cytoplasm [46]. In addition, the miRNAs transferred by exosomes also have biological activation and can be targeted and regulated by the level of mRNA in cells after entering the target cells. As a cell-cell communication medium, exosomes act on target cells in three ways: the ligands on the surface of exosomes directly bind to the receptor of target cells; the soluble components of proteins on the membrane hydrolyzed by protease bind to the receptor of target cells, and they act through the fusion or endocytosis of target cells. The function of recognizing target cells by exosomes is to avoid being randomly transmitted to other tissue cells to play a targeted therapeutic role. It can provide drug carriers for precision medicine and horizontally transfer the contents to target cells through membrane fusion to play a biological function $[47,48]$.

The exosomes can fuse directly with the target cell membrane and release the proteins, mRNA, and miRNAs. MicroRNA is a non-coding RNA that mediates 
Table $2 \mathrm{GO}$ analysis of the candidate miRNAs in TRD

\begin{tabular}{|c|c|c|}
\hline Term & Overlap & $P$-values \\
\hline Axon part (GO:0033267) & $32 / 415$ & 0.003757 \\
\hline CHD-type complex (GO:0090545) & $4 / 18$ & 0.008459 \\
\hline Cytoplasmic ribonucleoprotein granule (GO:0036464) & $23 / 268$ & 0.003642 \\
\hline Early endosome (GO:0005769) & $34 / 434$ & 0.002231 \\
\hline NuRD complex (GO:0016581) & $4 / 18$ & 0.008459 \\
\hline PML body (GO:0016605) & $13 / 113$ & 0.002301 \\
\hline Postsynaptic density (GO:0014069) & $25 / 35$ & 0.006811 \\
\hline Ribonucleoprotein granule (GO:0035770) & $23 / 281$ & 0.006435 \\
\hline Transcription factor complex (GO:0005667) & $33 / 403$ & 0.001257 \\
\hline Transcriptional repressor complex (GO:0017053) & $11 / 90$ & 0.003031 \\
\hline Appendage development (GO:0048736) & $23 / 188$ & 0.000026 \\
\hline Axonogenesis (GO:0007409) & $46 / 498$ & 0.000010 \\
\hline Cell fate commitment (GO:0045165) & $34 / 304$ & 0.000003 \\
\hline Embryonic organ development (GO:0048568) & $43 / 480$ & 0.000041 \\
\hline Limb development (GO:0060173) & $23 / 188$ & 0.000026 \\
\hline Lung development (GO:0030324) & 23/193 & 0.000040 \\
\hline Positive regulation of neuron differentiation (GO:0045666) & $41 / 402$ & 0.000003 \\
\hline Positive regulation of neuron projection development (GO:0010976) & $32 / 304$ & 0.000018 \\
\hline Regulation of cellular component size (GO:0032535) & $39 / 407$ & 0.000021 \\
\hline Regulation of transcription involved in cell fate commitment (GO:0060850) & $7 / 18$ & 0.000010 \\
\hline DNA-binding transcription activator activity, RNA polymerase II-specific (GO:0001228) & $46 / 479$ & 0.000009 \\
\hline GTP binding (GO:0005525) & $33 / 406$ & 0.002841 \\
\hline Guanyl nucleotide binding (GO:0019001) & $35 / 429$ & 0.002028 \\
\hline Guanyl ribonucleotide binding (GO:0032561) & $35 / 429$ & 0.002028 \\
\hline Nucleoside binding (GO:0001882) & $33 / 422$ & 0.005119 \\
\hline Purine nucleoside binding (GO:0001883) & $33 / 414$ & 0.003839 \\
\hline Purine ribonucleoside binding (GO:0032550) & $33 / 411$ & 0.003434 \\
\hline Ribonucleoside binding (GO:0032549) & $33 / 415$ & 0.003982 \\
\hline SUMO transferase activity (GO:0019789) & $5 / 20$ & 0.002265 \\
\hline Transcription corepressor activity (GO:0003714) & $22 / 195$ & 0.000214 \\
\hline
\end{tabular}

Table 3 KEGG analysis of the candidate miRNAs in TRD

\begin{tabular}{lll}
\hline ID Description & Overlap & $P$-values \\
\hline Steroid biosynthesis (KEGG:hsa00100) & $13 / 20$ & $2.156 \mathrm{E}-07$ \\
Parathyroid hormone synthesis, secretion, and action (KEGG:hsa04928) & $35 / 106$ & $4.265 \mathrm{E}-07$ \\
PI3K-Akt signaling pathway (KEGG:hsa04151) & $77 / 354$ & $3.086 \mathrm{E}-05$ \\
Ras signaling pathway (KEGG:hsa04014) & $55 / 232$ & $3.744 \mathrm{E}-05$ \\
Breast cancer (KEGG:hsa05224) & $38 / 147$ & $8.661 \mathrm{E}-05$ \\
MAPK signaling pathway (KEGG:hsa04010) & $64 / 294$ & 0.0001402 \\
Calcium signaling pathway (KEGG:hsa04020) & $54 / 240$ & 0.0001912 \\
Complement and coagulation cascades (KEGG:hsa04610) & $24 / 85$ & 0.000424 \\
Chemokine signaling pathway (KEGG:hsa04062) & $44 / 192$ & 0.000483 \\
Cytokine-cytokine receptor interaction (KEGG:hsa04060) & $62 / 295$ & 0.0004842 \\
\hline
\end{tabular}




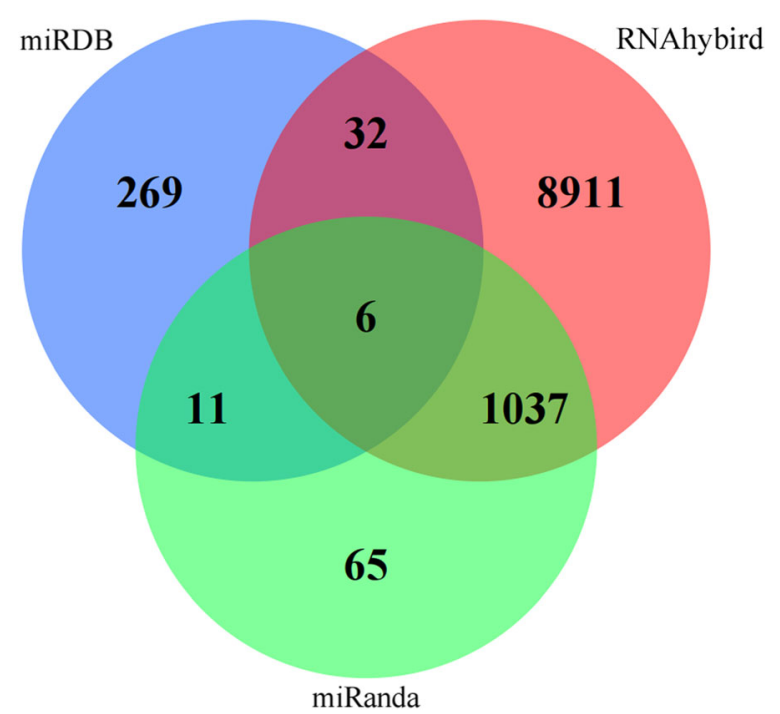

Fig. 3 Venn diagram of target gene prediction results. This figure shows the gene prediction results by three different methods (miRanda, RNAhybrid, and miRDB). The result of miRDB is superimposed in blue. The result of RNAhybrid is superimposed in red. The result of miRanda is superimposed in green. The overlapping shows the same result in 2 or 3 different methods

post-transcriptional silencing of mRNA and can regulate various cellular and molecular signaling pathways. The miRNA is widely found in human blood, urine, CSF, and other body fluid samples and provides abundant, stable, sensitive, and specific biological information, which can transfer information in the circulatory system. In recent years, many studies have shown that miRNA is one of the important molecules in the pathogenesis of depression. It has been demonstrated that the deregulation of
miR-16, miR-451, miR-223, miR-182, and other miRNAs may be related to the depression state of different patients [49]. These findings suggest that tissue-specific circulating miRNAs can be used as biomarkers to diagnose and treat depression. At present, the research methods of miRNA are mainly through real-time quantitative PCR and gene chip technology. These methods mainly focus on the expression and quantification of miRNA and are limited to the research of miRNA with sequence information or secondary stem ring structure information, so it is impossible to find new miRNA molecules. Based on high-throughput sequencing technology, which enables researchers to sequence miRNAs in samples with high-throughput directly, higher sensitivity, large sequencing throughput, and be able to detect rare transcripts with extremely low abundance, and it can annotate known miRNAs with a public miRNA database, and further analyze unmatched new miRNA species and isomers, and sought further research information [50, 51].

In this study, we used high-throughput sequencing technology to screen sensitive miRNAs related to depression. The results showed that the exoRNeasy serum/ plasma MIDI kit was successfully used to extract plasma exosomes. Fifteen miRNAs with significant expression differences were screened by high-throughput sequencing, among which miR-19a-3p, miR-144-3p, miR-130a3p, miR-355-3p, miR-101-3p, miR-1277-5p, miR-15a-5p, miR-29c-3p, and miR-21-5p were upregulated, miR7704, miR-1292-3p, miR-1909-5p, miR-5001-5p and miR-4688 were downregulated. Some of these abnormal miRNAs have been reported. For example, miR-144-3p is downregulated in the plasma of patients with

Table 4 Putative target genes of the differentially expressed miRNAs in plasma-derived exosomes in four patients with TRD compared to four healthy control subjects

\begin{tabular}{|c|c|c|c|c|}
\hline Genes & NO. of miRNAs targeted & miRNA expression & Reference(s) & Function(s) \\
\hline ADRA1B & 1 (has-miR-1292-3p) & Down & [33] & GABA, serotonin \\
\hline ADRB1 & 1 (has-miR-1292-3p) & Down & {$[34]$} & Synapsin \\
\hline CACNA1C & 1 (has-miR-1292-3p) & Down & [35] & Synapsin Dopamine Glutamate Adrenergic \\
\hline CREB1 & 1 (has-miR-1292-3p) & Down & {$[36]$} & Synapsin Dopamine NMDA Glutamate serotonin \\
\hline FGFR1 & 1 (has-miR-1292-3p) & Down & {$[37]$} & Dopamine \\
\hline GNAS & 1 (has-miR-1292-3p) & Down & {$[38]$} & Synapsin Glutamate serotonin \\
\hline GRIK5 & 1 (has-miR-1292-3p) & Down & {$[33]$} & Synapsin Dopamine NMDA Glutamate GABA \\
\hline HTR1A & 1 (has-miR-1292-3p) & Down & [39] & Synapsin Glutamate \\
\hline NOS1 & 1 (has-miR-1292-3p) & Down & {$[40]$} & Serotonin Dopamine NMDA Glutamate GABA Adrenergic \\
\hline OPRM1 & 1 (has-miR-1292-3p) & Down & [41] & Synapsin Dopamine Glutamate GABA Adrenergic \\
\hline$P 2 R \times 7$ & 1 (has-miR-1292-3p) & Down & {$[42]$} & Glutamate GABA \\
\hline PDE10A & 1 (has-miR-1292-3p) & Down & {$[43]$} & Synapsin \\
\hline NUFIP2 & 1 (has-miR-335-5p) & Up & {$[44]$} & MDD \\
\hline TCF4 & 1 (has-miR-335-5p) & Up & {$[44]$} & MDD \\
\hline
\end{tabular}


depression, and the expression level of miR-144-3p in the plasma is related to depression symptoms [52]. Some studies have shown that miR-144-3p can target the mRNA of glutamate decarboxylase-67 (GAD67), vesicular $\gamma$-aminobutyric acid (GABA) transporter, and GABA transporter-3; inhibit their translation; and thus destroy the release and uptake of GABA. This may constitute the subcellular and molecular mechanism of GABA reduction in patients with depression [53]. The miR-335 participates in treating severe depression by targeting glutamate metabotropic receptor 4 (GRM4) [54]. miR$15 \mathrm{~b}$ is upregulated in the medial prefrontal cortex of depression like mice [55]. miR-101 is decreased in the ventrolateral orbital cortex (VLOs) of chronic unpredictable mild stress (CUMS) rat brain and targets dualspecific phosphatase 1 (DUSP1) to modulate depressivelike behaviors [56]. miR-101 and miR-130a were significantly downregulated in the prefrontal cortex of depressed suicide subjects [57]. These results all prove that microRNAs play a key role in the pathogenesis of depression by targeting multiple cellular and molecular pathways. We further used unpaired Student's $t$ test with Welch's correction to analyze the expression levels of these 15 microRNAs and found that has-miR-335 and has-miR-1292 still had statistical differences. These two microRNAs have been proved to be associated with synaptic function and TRD.

Notably, compared with plasma, urine, or other biological fluids, exosomes are rich in microRNA (miRNA). Most miRNAs that can be obtained from serum and saliva are contained in exosomes, and some miRNAs seem to depend on exosomes because they are not directly detected as free-floating molecules in biological fluids [13]. However, there are still few studies on exosomal microRNAs in depression. Wei et al. explored serum exosome miRNA expression profile in patients with MDD and healthy control subjects to identify potential MDD markers by microRNA sequencing. Compared with control subjects, 24 microRNAs were upregulated and 14 were downregulated. The upregulation of miR-19a-3p was consistent with our test results [20].

$\mathrm{GO}$ analysis showed that the target genes of different miRNAs were mainly enriched in the postsynaptic density and axonogenesis. The changes and damages of neural plasticity were closely related to the development of depression. The decrease of synaptic formation, which is closely related to the impairment of synaptic connections between neurons [58], is related to the pathogenesis of adult depression [59]. In the KEGG pathway analysis, the PI3K-Akt signaling pathway, MAPK signaling pathway, and Ras signaling pathway are closely related to depression. MAPK signaling pathway and Ras signaling pathway are some of the downstream pathways of BDNF [60]. Brain-derived neurotrophic factor (BDNF) and its receptor are widely expressed in the CNS, which affect many functions of neurons, such as the growth, morphology, and plasticity of synaptic structure [61]. The BDNF related signaling pathway is one of the pathogenesis of depression. They exist in neurons and other brain cells and participate in growth factors, cytokines, oxidative stress, hormones, and other physical stimuli [62]. A PI3K-Akt signaling pathway is downstream of the BDNF-TrkB signaling pathway. Some studies have shown that the PI3K-Akt signaling pathway is abnormal in depression, and it plays an important role in antidepressant and synaptic protein synthesis $[63,64]$. In addition, target genes selected based on differential expression of microRNAs are involved in the synaptic synthesis and transport of many neurotransmitters, including dopamine, glutamate, norepinephrine, serotonin, and the binding process with corresponding receptors [29]. A genome-wide association meta-analysis based on 135,458 cases and 344,901 controls and identified 44 independent and significant loci, including NUFIP2 and TCF4. MicroRNAs in exosomes may regulate the release and function of neurotransmitters closely related to depression by regulating synaptic terminals, affecting the function of different brain regions, and regulating depression behavior. All the above pieces of evidence indicated the potential role of exosomal miRNA in depression.

\section{Conclusion}

In conclusion, exosomal miRNAs can regulate postsynaptic density and axonogenesis in TRD. Further studies will evaluate whether the dysregulation of any specific miRNA or combination of miRNAs has a role in the pathology of depression or whether they may represent potential markers of the disease or treatment response. In addition to the traditional direct regulation of gene transcription level, miRNAs in exosomes are closely related to the function of neuronal synapses and neurotransmitters and may be involved in regulating neurotransmitter release between different brain regions.

\section{Supplementary Information}

The online version contains supplementary material available at https://doi. org/10.1186/s40246-021-00354-z.

Additional file 1: Supplement Table 1.

Additional file 2: Supplement Figure 1.

Acknowledgements

This work was supported by the National Natural Science Foundation of China (No. 81871065 and 82071525 to Q-G. Z).

Authors' contributions

$J Z, Q G-Z$, and FH designed the study and wrote the protocol. LD-L, ZW-D, MN, KG, FM, HC-D, YP-Z, CW, and LL-W performed the statistical analyses. All the authors made a significant contribution to the interpretation of the data 
and drafting the manuscript and/or revising it critically for intellectual content. All authors have approved the final version of the manuscript.

\section{Availability of data and materials}

Data should be made available from corresponding authors on reasonable request.

\section{Declarations}

Ethics approval and consent to participate

The study was approved by the ethics board at Nanjing First Hospital Organization in Nanjing, China.

\section{Competing interests}

All authors declare that they have no competing financial interests.

\section{Author details}

'Department of Clinical Pharmacology, School of Pharmacy, Nanjing Medical University, Nanjing 211166, Jiangsu Province, China. ${ }^{2}$ Nanjing Brain Hospital Affiliated to Nanjing Medical University, Nanjing 210029, Jiangsu Province, China. ${ }^{3}$ Functional Brain Imaging Institute of Nanjing Medical University, Nanjing 210029, Jiangsu Province, China. ${ }^{4}$ Sir Run Run Hospital, Nanjing Medical University, Nanjing 211167, Jiangsu Province, China. ${ }^{5}$ Key Laboratory of Cardiovascular and Cerebrovascular Medicine, School of Pharmacy, Nanjing Medical University, Nanjing 211166, Jiangsu Province, China.

Received: 12 April 2021 Accepted: 2 August 2021

Published online: 21 August 2021

\section{References}

1. Malhi GS, Mann JJ. Depression. Lancet. 2018;392(10161):2299-312. https:// doi.org/10.1016/S0140-6736(18)31948-2.

2. Hamon M, Blier P. Monoamine neurocircuitry in depression and strategies for new treatments. Prog Neuro-Psychopharmacol Biol Psychiatry. 2013;45: 54-63. https://doi.org/10.1016/j.pnpbp.2013.04.009.

3. Juruena MF, Bocharova M, Agustini B, Young AH. Atypical depression and non-atypical depression: Is HPA axis function a biomarker? A systematic review. J Affect Disord. 2018;233:45-67. https://doi.org/10.1016/j.jad.2017.09. 052.

4. Kim YK, Na KS, Myint AM, Leonard BE. The role of pro-inflammatory cytokines in neuroinflammation, neurogenesis and the neuroendocrine system in major depression. Prog Neuro-Psychopharmacol Biol Psychiatry. 2016;64:277-84. https://doi.org/10.1016/j.pnpbp.2015.06.008.

5. Duman RS, Sanacora G, Krystal JH. Altered connectivity in depression: GABA and glutamate neurotransmitter deficits and reversal by novel treatments. neuron. 2019;102(1):75-90. https://doi.org/10.1016/j.neuron.2019.03.013.

6. Halaris A, Sohl E, Whitham EA. Treatment-resistant depression revisited: a glimmer of hope. J Pers Med. 2021;11(2):155. https://doi.org/10.3390/jpm11 020155.

7. Raposo G, Stoorvogel W. Extracellular vesicles: exosomes, microvesicles, and friends. J Cell Biol. 2013;200(4):373-83. https://doi.org/10.1083/jcb.20121113 8.

8. Mustapic M, Eitan E, Werner JK Jr, Berkowitz ST, Lazaropoulos MP, Tran J, et al. Plasma extracellular vesicles enriched for neuronal origin: a potential window into brain pathologic processes. Front Neurosci. 2017;11:278. https://doi.org/10.3389/fnins.2017.00278.

9. Tsilioni I, Panagiotidou S, Theoharides TC. Exosomes in neurologic and psychiatric disorders. Clin Ther. 2014;36(6):882-8. https://doi.org/10.1016/j. clinthera.2014.05.005

10. Luarte A, et al. Astrocytes at the hub of the stress response: potential modulation of neurogenesis by miRNAs in astrocytederived exosomes. Stem Cells Int. 2017;2017:1719050. https://doi.org/10.1155/2017/1719050

11. Bátiz LF, et al. Exosomes as novel regulators of adult neurogenic niches. Front Cell Neurosci. 2015;9:501. https://doi.org/10.3389/fncel.2015.00501.

12. Lafourcade C, Ramírez JP, Luarte A, Fernández A, Wyneken U. MiRNAs in astrocyte-derived exosomes as possible mediators of neuronal plasticity. J Exp Neurosci. 2016;10(Suppl 1):1-9. https://doi.org/10.4137/JEN.S39916.

13. Gallo A, Tandon M, Alevizos I, Illei GG. The majority of microRNAs detectable in serum and saliva is concentrated in exosomes. PLoS One. 2012;7(3): e30679. https://doi.org/10.1371/journal.pone.0030679.
14. Dwivedi Y. Emerging role of microRNAs in major depressive disorder: diagnosis and therapeutic implications. Dialogues Clin Neurosci. 2014;16(1): 43-61.

15. Saeedi $\mathrm{S}$, et al. The emerging role of exosomes in mental disorders. 2019; 9(1):122. https://doi.org/10.1038/s41398-019-0459-9.

16. Banigan MG, Kao PF, Kozubek JA, Winslow AR, Medina J, Costa J, et al. Differential expression of exosomal microRNAs in prefrontal cortices of schizophrenia and bipolar disorder patients. PLoS One. 2013;8(1):e48814 https://doi.org/10.1371/journal.pone.0048814.

17. Du Y, et al. Genome-wide, integrative analysis implicates exosome-derived microRNA dysregulation in schizophrenia. Schizophr Bull. 2019;45(6):125766. https://doi.org/10.1093/schbul/sby191.

18. Sakamoto $\mathrm{S}$, et al. Alterations in circulating extracellular vesicles underlie social stress-induced behaviors in mice. FEBS Open Bio. 2021. https://doi. org/10.1002/2211-5463.13204.

19. Liang JQ, Liao HR, Xu CX, Li XL, Wei ZX, Xie GJ, et al. Serum exosomederived miR-139-5p as a potential biomarker for major depressive disorder. Neuropsychiatr Dis Treat. 2020;16:2689-93. https://doi.org/10.2147/NDT.S2 77392.

20. Wei ZX, Xie GJ, Mao X, Zou XP, Liao YJ, Liu QS, et al. Exosomes from patients with major depression cause depressive-like behaviors in mice with involvement of miR-139-5p-regulated neurogenesis.

Neuropsychopharmacology. 2020;45(6):1050-8. https://doi.org/10.1038/s413 86-020-0622-2.

21. Guo H, Huang B, Wang $Y$, Zhang $Y$, Ma Q, Ren $Y$. Bone marrow mesenchymal stem cells-derived exosomes improve injury of hippocampal neurons in rats with depression by upregulating microRNA-26a expression. Int Immunopharmacol. 2020;82:106285. https://doi.org/10.1016/j.intimp.202 0.106285 .

22. Li D, Wang Y, Jin X, Hu D, Xia C, Xu H, et al. NK cell-derived exosomes carry miR-207 and alleviate depression-like symptoms in mice. J

Neuroinflammation. 2020;17(1):126. https://doi.org/10.1186/s12974-020-01 787-4.

23. Alvarez-Erviti L, Seow Y, Yin HF, Betts C, Lakhal S, Wood MJA. Delivery of siRNA to the mouse brain by systemic injection of targeted exosomes. Nat Biotechnol. 2011;29(4):341-5. https://doi.org/10.1038/nbt.1807.

24. Zimmerman M, Martinez JH, Young D, Chelminski I, Dalrymple K. Severity classification on the Hamilton Depression Rating Scale. J Affect Disord. 2013; 150(2):384-8. https://doi.org/10.1016/j.jad.2013.04.028.

25. Hafner M, Landgraf $P$, Ludwig J, Rice A, Ojo T, Lin C, et al. Identification of microRNAs and other small regulatory RNAs using CDNA library sequencing. Methods. 2008;44(1):3-12. https://doi.org/10.1016/j.ymeth.2007.09.009.

26. Robinson MD, McCarthy DJ, Smyth GK. edgeR: a Bioconductor package for differential expression analysis of digital gene expression data. Bioinformatics. 2010;26(1):139-40. https://doi.org/10.1093/bioinformatics/ btp616.

27. Quinlan AR, Hall IM. BEDTools: a flexible suite of utilities for comparing genomic features. Bioinformatics. 2010;26(6):841-2. https://doi.org/10.1093/ bioinformatics/btq033.

28. Liang J, LV J, Liu Z. Identification of stage-specific biomarkers in lung adenocarcinoma based on RNA-seq data. Tumour Biol. 2015;36(8):6391-9. https://doi.org/10.1007/s13277-015-3327-0.

29. Nahm KY, et al. Gene profiling of bone around orthodontic mini-implants by RNA-sequencing analysis. Biomed Res Int. 2015;2015:538080. https://doi. org/10.1155/2015/538080.

30. Théry $C$, et al. Isolation and characterization of exosomes from cell culture supernatants and biological fluids. Curr Protoc Cell Biol. 2006. Chapter 3: p. Unit 3.22. https://doi.org/10.1002/0471143030.cb0322s30.

31. Huang G, Cao M, Huang Z, Xiang Y, Liu J, Wang Y, et al. Small RNAsequencing identified the potential roles of neuron differentiation and MAPK signaling pathway in dilated cardiomyopathy. Biomed Pharmacother. 2019;114:108826. https://doi.org/10.1016/j.biopha.2019.108826.

32. Khan HA, Zhao Y, Wang L, Li Q, du YA, Dan Y, et al. Identification of miRNAs during mouse postnatal ovarian development and superovulation. J Ovarian Res. 2015;8(1):44. https://doi.org/10.1186/s13048-015-0170-2.

33. Sun $X$, Zhou $M$, Wen $G$, Huang $Y$, Wu J, Peng $L$, et al. Paroxetine Attenuates Cardiac Hypertrophy Via Blocking GRK2 and ADRB1 Interaction in Hypertension. J Am Heart Assoc. 2021;10(1):e016364. https://doi.org/10.11 61/JAHA.120.016364

34. Lee PH, Perlis RH, Jung JY, Byrne EM, Rueckert E, Siburian $\mathrm{R}$, et al. Multi-locus genome-wide association analysis supports the role of glutamatergic 
synaptic transmission in the etiology of major depressive disorder. Trans Psychiatry. 2012;2(11):e184. https://doi.org/10.1038/tp.2012.95.

35. Calabrò M, Mandelli L, Crisafulli C, Lee SJ, Jun TY, Wang SM, et al. Genes involved in neurodevelopment, neuroplasticity and major depression: no association for CACNA1C, CHRNA7 and MAPK1. Clin Psychopharmacol Neurosci. 2019;17(3):364-8. https://doi.org/10.9758/cpn.2019.17.3.364

36. Xiao X, Zhang C, Grigoroiu-Serbanescu M, Wang L, Li L, Zhou D, et al. The CAMP responsive element-binding (CREB)-1 gene increases risk of major psychiatric disorders. Mol Psychiatry. 2018;23(9):1957-67. https://doi.org/10.1 038/mp.2017.243.

37. Salmaso N, Stevens HE, McNeill J, ElSayed M, Ren Q, Maragnoli ME, et al. Fibroblast growth factor 2 modulates hypothalamic pituitary axis activity and anxiety behavior through glucocorticoid receptors. Biol Psychiatry. 2016;80(6):479-89. https://doi.org/10.1016/j.biopsych.2016.02.026.

38. Amare AT, Schubert KO, Klingler-Hoffmann M, Cohen-Woods S, Baune BT. The genetic overlap between mood disorders and cardiometabolic diseases: a systematic review of genome wide and candidate gene studies. Trans Psychiatry. 2017;7(1):e1007. https://doi.org/10.1038/tp.2016.261.

39. Badamasi IM, Lye MS, Ibrahim N, Abdul Razaq NA, Ling KH, Stanslas J. Serotonergic receptor gene polymorphism and response to selective serotonin reuptake inhibitors in ethnic Malay patients with first episode of major depressive disorder. Pharm J. 2021;21(4):498-509. https://doi.org/10.1 038/s41397-021-00228-6.

40. Wigner $P$, et al. The effect of chronic mild stress and escitalopram on the expression and methylation levels of genes involved in the oxidative and nitrosative stresses as well as tryptophan catabolites pathway in the blood and brain structures. Int J Mol Sci. 2020;22(1):10. https://doi.org/10.3390/ ijms22010010.

41. Grunebaum MF, Galfalvy HC, Liu J, Huang YY, Marcott S, Burke AK, et al. Opioid receptor $\mu-1$ and ketamine effects in a suicidal depression trial: a post hoc exploration. J Clin Psychopharmacol. 2020;40(4):420-2. https://doi. org/10.1097/JCP.0000000000001237.

42. Kristof Z, Eszlari N, Sutori S, Gal Z, Torok D, Baksa D, et al. P2RX7 gene variation mediates the effect of childhood adversity and recent stress on the severity of depressive symptoms. PLoS One. 2021;16(6):e0252766. https://doi.org/10.1371/journal.pone.0252766.

43. Delhaye S, Bardoni B. Role of phosphodiesterases in the pathophysiology of neurodevelopmental disorders. Mol Psychiatry. 2021. https://doi.org/10.103 8/s41380-020-00997-9.

44. Wray NR, et al. Genome-wide association analyses identify 44 risk variants and refine the genetic architecture of major depression. Nat Genet. 2018; 50(5):668-81. https://doi.org/10.1038/s41588-018-0090-3.

45. Pan BT, Johnstone RM. Fate of the transferrin receptor during maturation of sheep reticulocytes in vitro: selective externalization of the receptor. Cell. 1983;33(3):967-78. https://doi.org/10.1016/0092-8674(83)90040-5.

46. Valadi H, Ekström K, Bossios A, Sjöstrand M, Lee JJ, Lötvall JO. Exosomemediated transfer of mRNAs and microRNAs is a novel mechanism of genetic exchange between cells. Nat Cell Biol. 2007;9(6):654-9. https://doi. org/10.1038/ncb1596.

47. De Toro J, et al. Emerging roles of exosomes in normal and pathological conditions: new insights for diagnosis and therapeutic applications. Front Immunol. 2015;6:203.

48. Keller S, Sanderson MP, Stoeck A, Altevogt P. Exosomes: from biogenesis and secretion to biological function. Immunol Lett. 2006;107(2):102-8. https://doi.org/10.1016/j.imlet.2006.09.005.

49. Issler O, Chen A. Determining the role of microRNAs in psychiatric disorders. Nat Rev Neurosci. 2015;16(4):201-12. https://doi.org/10.1038/nrn3879.

50. Zhu H, Urban DJ, Blashka J, McPheeters MT, Kroeze WK, Mieczkowski P, et al. Quantitative analysis of focused a-to-I RNA editing sites by ultra-highthroughput sequencing in psychiatric disorders. PLoS One. 2012;7(8):e43227. https://doi.org/10.1371/journal.pone.0043227.

51. Hansen KF, et al. Profiling status epilepticus-induced changes in hippocampal RNA expression using high-throughput RNA sequencing. Sci Rep. 2014:4:6930.

52. Wang $X$, Sundquist $K$, Hedelius A, Palmér K, Memon AA, Sundquist J. Circulating microRNA-144-5p is associated with depressive disorders. Clin Epigenetics. 2015;7(1):69. https://doi.org/10.1186/s13148-015-0099-8.

53. Ma K, Xu A, Cui S, Sun MR, Xue YC, Wang JH. Impaired GABA synthesis, uptake and release are associated with depression-like behaviors induced by chronic mild stress. Transl Psychiatry. 2016;6(10):e910. https://doi.org/10.1 038/tp.2016.181.
54. Schmidt K, Cowen PJ, Harmer CJ, Tzortzis G, Errington S, Burnet PWJ. Prebiotic intake reduces the waking cortisol response and alters emotional bias in healthy volunteers. Psychopharmacology. 2015;232(10):1793-801. https://doi.org/10.1007/s00213-014-3810-0.

55. Guo L, et al. microRNA-15b contributes to depression-like behavior in mice by affecting synaptic protein levels and function in the nucleus accumbens. J Biol Chem. 2020;295(20):6831-48. https://doi.org/10.1074/jbc.RA119.012047.

56. Zhao Y, et al. MicroRNA-101 in the ventrolateral orbital cortex (VLO) modulates depressive-like behaviors in rats and targets dual-specificity phosphatase 1 (DUSP1). Brain Res. 2017;1669:55-62. https://doi.org/10.1016/ j.brainres.2017.05.020.

57. Smalheiser NR, et al. MicroRNA expression is down-regulated and reorganized in prefrontal cortex of depressed suicide subjects. PLoS One. 2012;7(3):e33201. https://doi.org/10.1371/journal.pone.0033201.

58. Bambico FR, Belzung C. Novel insights into depression and antidepressants: a synergy between synaptogenesis and neurogenesis?. Curr Top Behav Neurosci. 2013;15:243-91. https://doi.org/10.1007/7854_2012_234.

59. Kuhn M, Höger N, Feige B, Blechert J, Normann C, Nissen C. Fear extinction as a model for synaptic plasticity in major depressive disorder. PLoS One. 2014;9(12):e115280. https://doi.org/10.1371/journal.pone.0115280.

60. Huang EJ, Reichardt LF. Trk receptors: roles in neuronal signal transduction. Annu Rev Biochem. 2003;72(1):609-42. https://doi.org/10.1146/annurev. biochem.72.121801.161629.

61. Park H, Poo MM. Neurotrophin regulation of neural circuit development and function. Nat Rev Neurosci. 2013;14(1):7-23. https://doi.org/10.1038/ nrn3379.

62. Chen P, Lin QS, Zhang LC. The distribution of MAP kinase phosphatase-1 in the cerebrospinal fluid-contacting nucleus and its functional contribution to depressive behaviors. Sheng Li Xue Bao. 2015;67(1):90-6.

63. Ludka FK, Constantino LC, Dal-Cim T, Binder LB, Zomkowski A, Rodrigues ALS, et al. Involvement of PI3K/Akt/GSK-3 $\beta$ and mTOR in the antidepressantlike effect of atorvastatin in mice. J Psychiatr Res. 2016;82:50-7. https://doi. org/10.1016/j.jpsychires.2016.07.004.

64. Budni J, Lobato KR, Binfaré RW, Freitas AE, Costa AP, Martín-de-Saavedra $M D$, et al. Involvement of PI3K, GSK-3 3 and PPARy in the antidepressant-like effect of folic acid in the forced swimming test in mice. J Psychopharmacol. 2012;26(5):714-23. https://doi.org/10.1177/0269881111424456.

\section{Publisher's Note}

Springer Nature remains neutral with regard to jurisdictional claims in published maps and institutional affiliations.

Ready to submit your research? Choose BMC and benefit from:

- fast, convenient online submission

- thorough peer review by experienced researchers in your field

- rapid publication on acceptance

- support for research data, including large and complex data types

- gold Open Access which fosters wider collaboration and increased citations

- maximum visibility for your research: over $100 \mathrm{M}$ website views per year

At BMC, research is always in progress.

Learn more biomedcentral.com/submissions 